\title{
Fingerprint Image Enhancement Based on a Half Gabor Filter
}

\author{
Wonchurl Jang, Deoksoo Park, Dongjae Lee, and Sung-jae Kim \\ Samsung Electronics, SoC R\&D Center, Korea \\ \{wc7.jang, deoksoo.park, djae.lee, sungjae.kim\}@samsung.com
}

\begin{abstract}
The performance of a fingerprint recognition system relies on the quality of the input fingerprint images. Several researches have been studied on the enhancement of fingerprint images for fingerprint recognition. The representative enhancement is the adaptive filtering method based on Gabor filter (GF). However, this method is computationally expensive due to the large mask size of GF. In this paper, we propose a half Gabor filter (HGF), which is suitable for fast implementation in spatial domain. The HGF is a modified filter which preserves the frequency property of a GF and reduces the mask size of the GF. Compared with the GF, the HGF not only reduces the processing time approximately by $41 \%$ but also enhances the fingerprint image which is as reliable as the GF.
\end{abstract}

Keywords: Gabor Filter, Gabor Enhancement, Fingerprint Image Enhancement, Adaptive Filter.

\section{Introduction}

Fingerprint patterns consist of ridges and valleys. These structures provide essential information for recognition. Conventionally, most fingerprint recognition systems use minutiae, a group of ridge end points and bifurcations, as the features of fingerprint patterns. The clearness of the extracted minutiae relies on the quality of the acquired fingerprint image. For this reason, fingerprint recognition systems heavily depend on the quality of the acquired fingerprint image. Hence, we need the image enhancing technique to improve the quality of the fingerprint image. Basically, the fingerprint image enhancement algorithm ought to satisfy two conditions. The first condition is to improve the clarity of ridge and valley structures of the fingerprint images. The second condition is to remove noise within ridge and valley pattern. The GF has the properties of spatial localization, orientation selectivity, and spatial-frequency selectivity [3]. With these properties, the GF satisfies the conditions of the fingerprint image enhancement algorithm [1]. Therefore the GF has been popularly used to enhance the fingerprint image. However this algorithm suffers from a major drawback which is a large computation cost. To solve this problem, we propose a HGF and a half Gabor stabilization filter (HGSF). The HGF is a modified filter which reduces the mask size of a GF and preserves the frequency property of a GF. The HGSF 
is a low pass filter which equalizes the frequency domain property of the HGF and GF. The proposed algorithm is faster than the conventional enhancement algorithm based on the GF and saves the memory space for filters. In addition, this algorithm extracts the ridge patterns as reliably as the GF.

\section{General Gabor Filter}

The GF has been used as a very useful tool to enhance a fingerprint image $[1,2,4]$. The configurations of the parallel ridges and valleys with well defined frequency and orientation in a fingerprint image provide useful information which helps removing the undesired noise. The sinusoidal-shaped waves of ridges and valleys vary slowly in a local constant orientation. Gabor filters have both frequencyselective and orientation-selective properties in frequency domain [2]. Therefore, it is appropriate to use GF as a bandpass filter to remove the noise and preserve true ridge/valley structures.

The 2-Dimensional GF is a harmonic oscillator, composed of a sinusoidal plane wave of a particular frequency and orientation within a Gaussian envelope. In [1], the general even-symmetric 2D GF is defined as

$$
\begin{aligned}
& h\left(x, y, \theta, f_{0}\right)=\exp \left\{-\frac{1}{2}\left[\frac{(x \cos \theta)^{2}}{\delta_{x}^{2}}+\frac{(y \sin \theta)^{2}}{\delta_{y}^{2}}\right]\right\} \cos \left(2 \pi f_{0} x_{\theta}\right) \\
& x_{\theta}=x \cos \theta+y \sin \theta
\end{aligned}
$$

where $\theta$ stands for an orientation of the GF and $f_{0}$ is the frequency of the sinusoidal plane wave (or the center frequency of the GF). Additionally, $\delta_{x}$ and $\delta_{y}$ represent the space constants of the Gaussian envelope along $x$ and $y$ axes. The frequency $f_{0}$ and the orientation $\theta$ are computed by a inter ridge distance and ridge orientation information [1].

\section{Half Gabor Filter and Fingerprint Image Enhancement}

In the previous section, we explained the general GF method based on the local ridge orientation and ridge frequency estimated from the input image. Although this algorithm can obtain a reliable ridge structures even for corrupted images, it is unsuitable for an embedded identification system because it spends a significant amount of efforts for GF computation. To improve the efficiency of GF, we propose the HGF and the HGSF algorithms. Figure 1 shows the block diagram (Fig. 1-b) of HGF and the image enhancement module based on HGF (Fig. 1-a). The frequency passband of HGF consists of the general GF term $G(u, v)$ and the phase shifted GF term $G(u-\pi, v-\pi)$. In order to reliably enhance the ridge patterns using the HGF, it is necessary to remove the noise passed by the phase shifted GF term and the filter mask normalization method to prevent the type changing of the enhanced ridge pattern. For this reason, we propose the HGSF, it is a low pass filter, which has a passband defined by the equation (13). Also, 


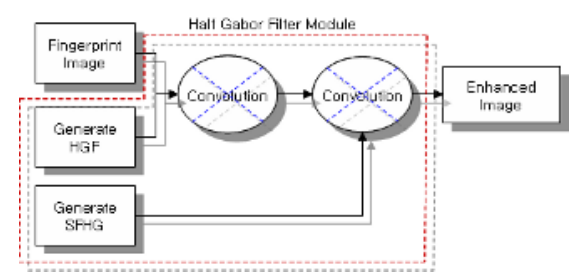

(a)

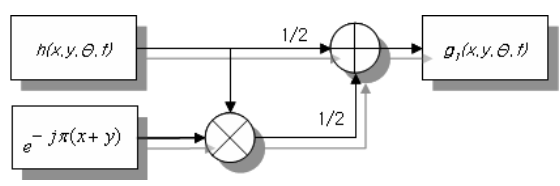

(b)

Fig. 1. Fingerprint image enhancement module based on the HGF: (a) Image enhancement module based on the HGF, (b) HGF generator

to prevent the type changing of the enhanced ridge pattern, we normalize the filter mask using the mask coefficient $\alpha(6)$.

The ridge pattern extraction steps are as follows :

Stage 1: Compute the mask coefficient $\alpha$ of HGF using the following equations.

$$
\begin{gathered}
p\left(x, y, \theta_{i}, f_{0}\right)= \begin{cases}h\left(x, y, \theta_{i}, f_{0}\right) & \text { if } h\left(x, y, \theta_{i}, f_{0}\right)>0 \\
0 & \text { otherwise. }\end{cases} \\
n\left(x, y, \theta_{i}, f_{0}\right)= \begin{cases}h\left(x, y, \theta_{i}, f_{0}\right) & \text { if } h\left(x, y, \theta_{i}, f_{0}\right)<0 \\
0 & \text { otherwise. }\end{cases} \\
\text { pSum }=\sum_{y=0}^{N-1} \sum_{x=0}^{N-1} p\left(x, y, \theta_{i}, f_{0}\right) \\
n \text { Sum }=\sum_{y=0}^{N-1} \sum_{x=0}^{N-1} n\left(x, y, \theta_{i}, f_{0}\right) \\
\alpha=\frac{|n S u m|}{p S u m}
\end{gathered}
$$

Here, $\theta_{i}$ is a quantified orientation $\left(\theta_{i}=0, \frac{\pi}{16}, \ldots, \frac{15 \pi}{16}\right)$, and $f_{0}$ is a local ridge frequency $\left(f_{0}=0.12\right)$.

Stage 2: Generate a half Gabor mask $g_{h}\left(x, y, \theta_{i}, f_{0}\right)$ of $N \times N(\mathrm{~N}=15)$ sizes. However the effective mask size of HGF is $N \times \frac{N}{2}$ because only non-zero elements are used. Figure 2 shows the masks of GF and HGF.

$$
\begin{gathered}
m\left(x, y, \theta_{i}, f_{0}\right)= \begin{cases}\alpha \cdot p\left(x, y, \theta_{i}, f_{0}\right) & \text { if } h\left(x, y, \theta_{i}, f_{0}\right)>0 \\
n\left(x, y, \theta_{i}, f_{0}\right) & \text { otherwise. }\end{cases} \\
g_{h}\left(x, y, \theta_{i}, f_{0}\right)=\frac{1}{2}\left\{m\left(x, y, \theta_{i}, f_{0}\right)+\left(e^{-j \pi}\right)^{x+y} m\left(x, y, \theta_{i}, f_{0}\right)\right\}
\end{gathered}
$$

Stage 3: Convolute a fingerprint image $t(x, y)$ with the HGF mask $g_{h}\left(x, y, \theta_{i}, f_{0}\right)$. We get the enhanced image $o(x, y)$. The discrete Fourier transform(DFT) of the image $o(x, y)$ is expressed by the $O(u, v)$. 


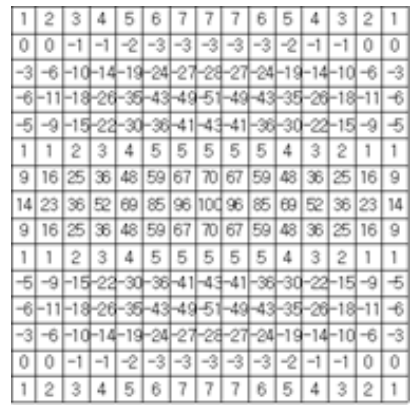

(a)

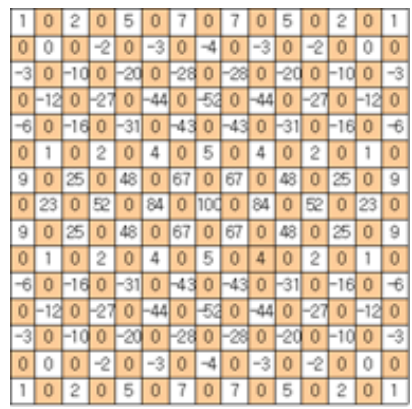

(b)

Fig. 2. Examples of the 15x15 GF's mask and the HGF's mask (for $\theta=0$ and $f=0.12$ ): (a) GF mask, (b) HGF mask (A coefficient in colored element is not an effective value)

$$
\begin{array}{r}
o(x, y)=\sum_{b=0}^{N-1} \sum_{a=0}^{N-1} g_{h}\left(a, b, \theta_{i}, f_{0}\right) \cdot t(a-x, b-y) \\
O(u, v)=T(u, v) \cdot \frac{1}{2}\left\{M\left(u, v, \theta_{i}, f_{0}\right)+M\left(u-\pi, v-\pi, \theta_{i}, f_{0}\right)\right\}
\end{array}
$$

where $T(u, v)$ and $M\left(u, v, \theta_{i}, f_{0}\right)$ are the DFT of $t(x, y)$ and $m\left(x, y, \theta_{i}, f_{0}\right)$.

Stage 4: Apply the HGSF $l(x, y)$ to an enhanced image $o(x, y)$.

$$
o_{l p f}(x, y)=\sum_{j=0}^{M-1} \sum_{i=0}^{M-1} l(i, j) \cdot o(i-x, j-y)
$$

where $\mathrm{l}(\mathrm{x}, \mathrm{y})$ is the $M \times M(M=3)$ sized gaussian filter having the passband which is defined by the equation (13).

Stage 5: Binarize the filtered image $o_{l p f}(x, y)$.

$$
b(x, y)= \begin{cases}1 & \text { if } o_{l p f}(x, y)>T_{b} \\ 0 & \text { otherwise. }\end{cases}
$$

From the stage 2, we generate a HGF mask which is half the size of GF mask. If we convolute a $S_{x} \times S_{y}$ sized fingerprint image with a $N \times N$ sized Gabor mask $h\left(x, y, \theta_{i}, f_{0}\right)$, then the computation power is $S_{x} \times S_{y} \times N \times N$. On the other hand, if we convolute a fingerprint image $S_{x} \times S_{y}$ with a $N \times N / 2$ sized half Gabor mask $g_{h}\left(x, y, \theta_{i}, f_{0}\right)$, then the computation power is $S_{x} \times S_{y} \times N \times N / 2$. The half Gabor filtered image $O(u, v)$ consists of the original GF passing image $I(u, v) H(u, v)$ and the phase shifted image $I(u, v) H(u-\pi, v-\pi)$, as shown in figure 3. To get an image such as the original Gabor filtered image, we have to remove the phase shifted image $I(u, v) H(u-\pi, v-\pi)$. For this reason, we apply the HGSF to the half Gabor filtered image. If the HGSF $l(x, y)$ satisfies the condition of the equation (13), then the olpf(x,y) is expressed by the general Gabor filtered image as the equation (14). 


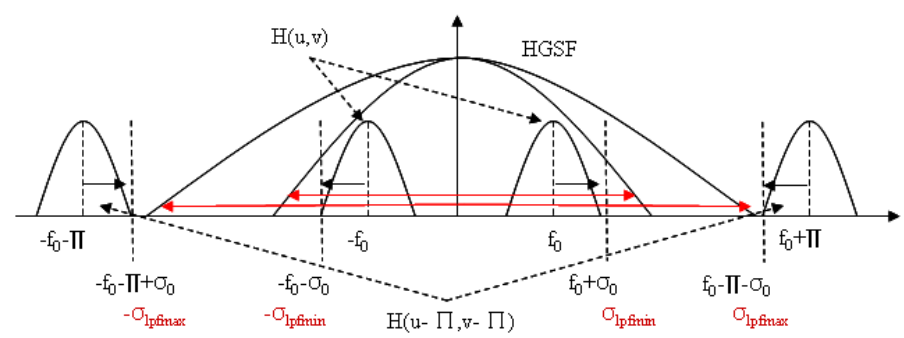

Fig. 3. The frequency property of HGF and the passband of HGSF

$$
\begin{gathered}
\sqrt{\left(f_{0}+\delta_{0}\right)^{2}}<\sqrt{\left(u^{2}+v^{2}\right)_{\max }}<\sqrt{\left(f_{0}+\pi-\delta_{0}\right)^{2}} \\
o_{l p f}(x, y)=i(x, y) \otimes \frac{1}{2} h(x, y, \theta, f)
\end{gathered}
$$

Where, $\delta_{0}=\delta_{x}=\delta_{y}\left(\delta_{0}=4.0\right), f_{0}$ is a ridge frequency $\left(f_{0}=0.12\right), \sqrt{\left(u^{2}+v^{2}\right)_{\max }}$ is bandwidth of $\mathrm{H}(\mathrm{u}, \mathrm{v})$.

\section{Experimental Results}

We evaluated the efficiency and robustness of our algorithm using FVC2002 Database1(DB2) and our collected fingerprint images (DB1), which were captured

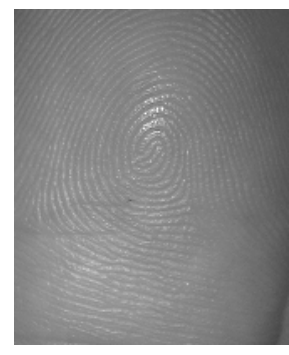

(a)

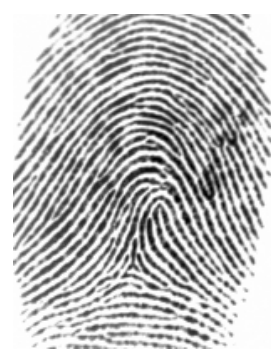

(d)

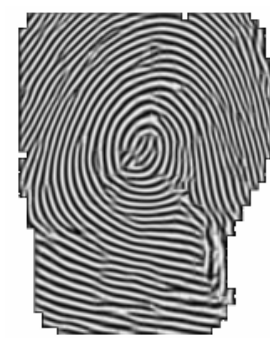

(b)

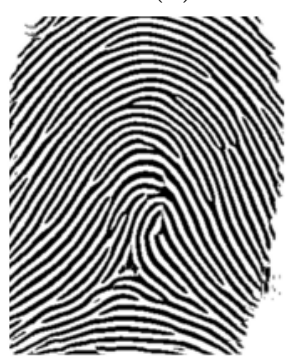

(e)

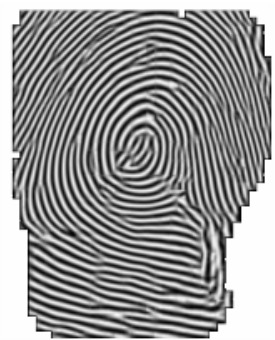

(c)

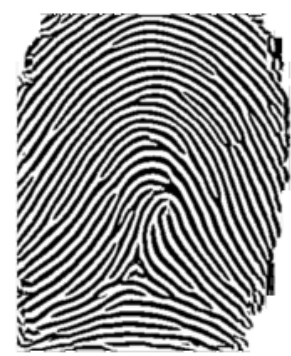

(f)

Fig. 4. Enhanced fingerprint images by a GF and HGF: (a) is a sample image of DB1 and (d) is a sample image of DB2; (b) and(e) are enhanced images by GF; (c) and(f) are enhanced images by HGF 
Table 1. The performance of minutiae extraction : DMR(Dropped minutiae ratio), $\operatorname{EMR}($ Exchanged minutiae ratio), TMR(True minutiae ratio), and FMR(False minutiae ratio)

\begin{tabular}{|c|c|c|c|c|c|c|c|c|}
\hline \multirow{2}{*}{ Filter } & \multicolumn{2}{|c|}{ DMR } & \multicolumn{2}{c|}{ EMR } & \multicolumn{2}{c|}{ FMR } & \multicolumn{2}{c|}{ TMR } \\
\cline { 2 - 9 } & DB1 & DB2 & DB1 & DB2 & DB1 & DB2 & DB1 & DB2 \\
\hline GF & $7 \%$ & $3 \%$ & $2 \%$ & $3 \%$ & $7 \%$ & $4 \%$ & $91 \%$ & $94 \%$ \\
\hline HGF & $8 \%$ & $3 \%$ & $2 \%$ & $5 \%$ & $9 \%$ & $5 \%$ & $90 \%$ & $92 \%$ \\
\hline
\end{tabular}

Table 2. The matching performance under the enhanced fingerprint images by HGF and GF

\begin{tabular}{|c|c|c|c|c|c|c|c|}
\hline \multirow{2}{*}{$\begin{array}{r}\text { Type } \\
\text { Filter }\end{array}$} & \multicolumn{3}{|c|}{ DB1 } & \multicolumn{3}{|c|}{ DB2 } \\
\cline { 3 - 8 } & & \multicolumn{2}{|c|}{ FAR } & EER & \multicolumn{2}{|c|}{ FAR } & EER \\
\cline { 3 - 8 } & & $0.1 \%$ & $1.0 \%$ & & $0.1 \%$ & $1.0 \%$ & \\
\hline GF & FRR & $5.24 \%$ & $2.78 \%$ & $2.32 \%$ & $3.38 \%$ & $1.53 \%$ & $1.25 \%$ \\
\hline HGF & FRR & $5.41 \%$ & $2.83 \%$ & $2.41 \%$ & $3.52 \%$ & $1.59 \%$ & $1.36 \%$ \\
\hline
\end{tabular}

Table 3. The time cost of image enhancement and the memory size for filter mask (Gabor orientations : 16 step, Gabor frequencies : 20 step, Gabor mask size : 15x15 pixels, Total number of Gabor masks : 320)

\begin{tabular}{|c|c|c|}
\hline Filter & Time Cost $(\mathrm{msec})$ & Memory Size (Kbyte) \\
\hline GF & 286 & 1033 \\
\hline HGF & 170 & 557 \\
\hline
\end{tabular}

by a 1.3 mega pixel digital camera. The DB2 consists of 840 fingerprint images (10 fingerprint-images are given by each 84 individuals) with various image qualities. Our experimental results show that our HGF is more efficient than the GF. Figure 4 shows the enhancement results obtained with the HGF and GF. In order to evaluate the performance, we examined the minutiae extracting rate, feature matching rate and time cost of the fingerprint image enhancement. In the examination of the minutiae extracting rate, we compared the minutiae manually taken by the experts with the minutiae automatically extracted using HGF and GF. Table 1 shows the minutiae extraction rate of HGF and GF. The difference between HGF and GF is less than $2 \%$ in TMR and FMR (Table 1). In the evaluation of matching performance, the difference between HGF and GF is less than about $0.1 \%$ in EER (Table 2). In the embedded system based on ARM-9, the GF takes $286 \mathrm{msec}$, but the HGF consumes 170 msec reducing $41 \%$ of time cost. Also, we can save the memory size for filter mask generation around $46 \%$ (Table 3 ).

\section{Conclusions}

Generally, the GF is used to enhance the fingerprint image. However the enhancement method based on the GF is computationally very expensive due to 
the large mask size. In this paper, we proposed an enhancement algorithm based on the HGF and HGSF which reliably improves the clarity of the ridge and valley patterns as well as permits a very efficient implementation in the spatial domain. We developed the HGF which reduces the mask size of the GF by using a frequency domain property of the GF in a fingerprint image. And we designed the HGSF which maintains a frequency domain property of the GF and HGF. The performance of our algorithm was evaluated using the minutiae extracting rate, feature matching rate, time cost and memory consumption. According to the experiment results, our algorithm is more suitable for an embedded system than the presented method based on the general GF.

\section{References}

1. L. Hong, Y. Wan, and A.K. Jain, Fingerprint Image Enhancement: Algorithm and Performance Evaluation, IEEE Trans. 1998, PAMI-20, (8), pp. 777-789 .

2. Chil-Jen Lee, Sheng-De Wang, and Kuo-Ping Wu, Fingerprint Recognition Using Principal Gabor Basis Function, Proceedings of 2001 International Symposium on Intelligent Multimedia, pp. 393-396.

3. J.G. Daugman, Uncertainty relation for resolution in space, spatial frequency, and orientation optimized by tow-dimensional visual cortical filter, J. Opt. Soc. Amer. A, vol. 2, no. 7, pp. 1160-1169, 1985.

4. Jianwei Yang, Lifeng Liu, Tianzi Jiang and Yong Fan, A modified Gabor filter design method for fingerprint image enhancement, Pattern Recognition Letters vol. 24, pp. 1805-1817, 2003. 